\title{
Some exact conditional tests for the multiplicative model to explain genotype-environment interaction
}

\author{
ROBERTO CRUZ MEDINA \\ Departamento de Matemáticas, Instituto Tecnológico de Sonora Cd. Obregón Son. C.P. 85000 México
}

\begin{abstract}
Genotype-environment interaction is often explained by the multiplicative model $\beta_{j} \varepsilon_{i}$ where $\beta_{j}$ is a parameter of the $j$-genotype not necessarily associated with the main effect for genotypes and $\varepsilon_{i}$ is the main effect for environment $i$. Mandel (1961) proposed a test for $H_{0}: \beta_{j}=0, j=1,2, \ldots g$; generalizing Tukey's 'One degree of freedom for non additivity'. Nevertheless if $H_{0}$ is rejected we do not have a method for detecting which $\beta_{j}$ is different from zero. In this paper a test for detecting which $\beta_{j}$ are different from zero when the general $H_{0}: \beta_{j}=0, j=1,2, \ldots g$; is rejected is proposed. In addition, if a within $(i, j)$-cell error is available another test to detect for which genotype the multiplicative model fails is proposed. These exact conditional tests can be useful to explain genotype-environment interaction.
\end{abstract}

Keywords: genotype-environment interaction, non-additivity, general linear model, two-factor interactions.

\section{Introduction}

Genotype-environment interaction is of major importance to the plant breeder in developing improved varieties because the relative rankings of varieties grown over a series of environments may differ statistically, causing problems in plant selection.

One of the most popular methods to explain the genotype-environment interaction seems to be the use of the multiplicative model. This method was first used by Mooers in 1921, as Westcott (1986) stated, but in most of the literature Yates \& Cochran (1938) appear as the first authors of this approach which consists of classifying genotypes by their regression coefficients calculated by regressing genotype means on environmental means.

Many authors show the statistical invalidity of using such regression analysis and their sums of squares but Shukla (1972), using the results of Mandel (1961) which are a generalization of that given by Tukey (1949), has shown that this method is statistically correct. If we reject the hypothesis $H_{0}: \beta_{j}=\beta_{j}$, with this method Shukla (1972) recommended an analysis including just two genotypes of interest to test the equality of any two $\beta_{j}$.
Let the model be

$$
\begin{aligned}
& Y_{i j}=\mu+\varepsilon_{i}+\tau_{j}+\gamma_{i j}+\bar{E}_{i j} \\
& Y_{i \cdot}=\Sigma_{i} Y_{i j} / g, Y_{j .}=\Sigma_{j} Y_{i j} / s, Y_{. .}=\Sigma Y_{i j} / s g
\end{aligned}
$$

where $Y_{i j}$ is the mean of $r$ replicates of the $j$ th genotype in the $i$ th environment, $\mu$ is the grand mean, $\varepsilon_{i}$ $(i=1, \ldots, s)$ the additive environment contribution of the $i$ th environment, $\tau_{j}(j=1, \ldots, g)$ the additive genetic contribution of the $j$ th genotype, $\gamma_{i j}$ the genotypeenvironment interaction of the $j$ th genotype in the $i$ th environment and $\bar{E}_{i j}$ are independent normal variables with mean zero and variance $\sigma^{2}$.

If we assume $\gamma_{i j}=\beta_{j} \varepsilon_{i}$, one way to estimate $\beta_{j}$ is first to obtain the estimates of $\mu, \varepsilon_{i}, \tau_{j}$ ignoring $\gamma_{i j}$ and then to adjust the residuals $Z_{i j}=Y_{i j}-Y_{i \cdot}-Y_{. j}+Y$.. to the multiplicative model using the estimates $e_{i}=Y_{i}-Y_{\text {. . of }}$ $\varepsilon_{i}$ the environmental effects. It is known that this procedure is not the least squares method (Gabriel, 1978) but it has proved to be useful.

Mandel (1961) proved that $H_{0}: \beta_{j}=0, j=1,2, \ldots, g$; can be tested with:

$F_{\mathrm{c}}=\frac{S S S /(g-1)}{S S R /(g-1)(s-2)}$ 
where:

$S S S=\Sigma_{j} S S S_{j}=\Sigma_{j} b_{j}^{2} \Sigma_{i} e_{i}^{2}$

$b_{j}=\Sigma_{i} e_{i} Z_{i j} / \Sigma e_{i}^{2}$

is the sum of squares of slopes and

$$
\begin{aligned}
S S D R & =S S R-S S S=\Sigma\left(Y_{i j}-Y_{i \cdot}-Y_{. j}+Y . .\right)^{2}-S S S \\
& =\Sigma_{j}\left(\Sigma_{i} Z_{i j}^{2}-b_{j}^{2} \Sigma e_{i}^{2}\right)=\Sigma_{j} S S D R_{j}
\end{aligned}
$$

is the sum of squares of residual, and $S S D R_{j}$ is the sum of squares of residual for genotype $j$. Eberhart \& Russell (1966) assigned $g(s-2)$ degrees of freedom to $S S D R$, the correct value is $(g-1)(s-2)$ then $F_{\mathrm{c}}$ will be distributed as $F$ on $(g-1)$ and $(g-1)(s-2)$ degrees of freedom.

\section{Tests for the multiplicative model}

In this section we will use the generalization of the linear model given by Milliken \& Graybill (1970), in which it is shown that some functions of estimable linear parameters can be included in a general linear model to explain residuals.

If we assume that interaction is not present $\left(\gamma_{i j}=0\right.$; $i=1, \ldots s ; j=1, \ldots g)$ we can split the $(s-1)(g-1)$ degrees of freedom of this factor into quadratic forms of the elements of the set of linear forms $\left\{e_{i}\right\}$ because they span two mutually orthogonal spaces (this can be seen in the analysis of variance table). Hence the conditional distribution of the $\left\{Z_{i j}\right\}$ given $\left\{e_{i}\right\}$, is identical to the unconditional distribution of the $\left\{Z_{i j}\right\}$ (Scheffé $p$. $133,1959)$, we can then postulate the model

$Z_{i j}=\beta_{j} e_{i}+d_{i j}+E_{i j}$

where $E_{i j}=\bar{E}_{i j}-\bar{E}_{i},-\bar{E}_{. j}+\bar{E}_{. .}$.

Observe that $\Sigma_{i} d_{i j}=0$ and $\Sigma_{j} d_{i j}=0$.

In order to develop the specific test for $H_{0}: \beta_{j}=\beta_{j}$, we will use a straightforward application of the general linear model theory.

Let us define:

$J_{v} \quad$ Matrix of $v \times v$ order consisting of ones.

$I_{v} \quad$ Identity matrix of order $v$.

$1_{v} \quad$ Column vector of $v$ ones.

$A^{\prime} \quad$ Transpose of matrix $A$.

$A B \quad$ Product of matrices $A$ and $B$.

$A \otimes B$ Direct product of matrices $A$ and $B$.

$$
\begin{aligned}
& e^{\prime}=\left(e_{1}, e_{2}, \ldots, e_{s}\right) \\
& \tau^{\prime}=\left(\tau_{1}, \tau_{2}, \ldots, \tau_{g}\right) \\
& \beta^{\prime}=\left(\beta_{1}, \beta_{2}, \ldots, \beta_{g}\right), \hat{\beta}^{\prime}=\left(b_{1}, b_{2}, \ldots, b_{g}\right) \\
& Z_{i}^{\prime}=\left(Z_{i 1}, Z_{i 2}, \ldots, Z_{i g}\right), Z^{\prime}=\left(Z_{1}^{\prime}, Z_{2}^{\prime}, \ldots, Z_{s}^{\prime}\right) .
\end{aligned}
$$

and $Y, \gamma, \bar{E}, E$ and $d$ in the same way as $Z$.

With these definitions it can be shown that:

$Z=H Y$ where $H$ is the centring matrix

$$
\begin{aligned}
H & =\left(I_{s}-J_{s} / s\right) \otimes\left(I_{g}-J_{g} / g\right) \\
& =I_{s} \otimes I_{g}-(1 / g) I_{s} \otimes J_{g}-(1 / s) J_{s} \otimes I_{g}+(1 / s g) J_{s} \otimes J_{g}
\end{aligned}
$$

then model (6) can be expressed by

$Z=e \otimes \beta+d+E$

or

$H Y=H e \otimes \beta+H(d+\bar{E})$.

If $\tau_{j}$ are taken as fixed effects we could assume $\Sigma_{j} \gamma_{i j}=0$ and then $\Sigma \beta_{j}=0$; in this case it can be proved that

$H e \otimes \beta=e \otimes \beta$.

\section{Tests for the coefficients of the model}

Under assumptions that $\bar{E}_{i j}$ are random independent normal variables with mean zero and variance $\sigma^{2}$, vector $E$ will have multinormal distribution with vector mean zero and covariance matrix $\operatorname{Cov}(E)=H \sigma^{2}$, that is $E \sim N\left(0, H \sigma^{2}\right)$ and then $Z \sim N\left(\mathrm{e} \otimes \beta, H \sigma^{2}\right)$. It can be proved that the conditional covariance matrix of $\hat{\beta}$ given $e_{i}$ is (see Appendix)

$\operatorname{Cov}(\hat{\beta})=\left(1 / g e^{\prime} e\right)\left(g I_{g}-J_{g}\right) \sigma^{2}$

$\operatorname{Var}\left(b_{j}\right)=\left(1 / g e^{\prime} e\right)(g-1) \sigma^{2}$.

If model (6) is correct an unbiased estimator of $\sigma^{2}$ is $\hat{\sigma}^{2}=S S D R /(g-1)(s-2)$ and the specific hypothesis

$H_{0}: \beta_{j}=0$ is rejected with $\alpha$ level if $\left|t_{c}\right| \geq t_{\alpha / 2}$ where

$t_{c}=b_{j} / \sqrt{\left(S S D R / g(s-2) e^{\prime} e\right)}$ or equivalently,

if $\left|b_{j}\right| \geq t_{\alpha / 2} \sqrt{\left(S S D R / g(s-2) e^{\prime} e\right)}$.

Hypothesis $H_{0}: \beta_{j}=\beta_{j}$, is rejected with $\alpha$ level if $\left|t_{c}\right| \geq t_{\alpha / 2}$

where $t_{c}=\left(b_{j}-b_{j^{\prime}}\right) / \sqrt{ }\left(2 S S D R /(g-1)(s-2) e^{\prime} e\right)$.

\section{Test to detect for which genotype the model fails}

In order to develop this test let

$Z_{j}^{\prime}=\left(Z_{1 j}, Z_{2 j}, \ldots, Z_{s j}\right)$

It can be seen from the covariance matrix of $Z$ that $\operatorname{Cov}\left(Z_{j}\right)=(g-1) g^{-1}\left(I_{s}-I_{s} / s\right) \sigma^{2}=M \sigma^{2}$.

Let

$$
\begin{aligned}
S S D_{j} & =g(g-1)^{-1}\left(\Sigma_{i} Z_{i j}^{2}-b_{j}^{2} e^{\prime} e\right) \\
& =g(g-1)^{-1}\left(S S R G_{j}-S S S_{j}\right)=g(g-1)^{-1} S S D R_{j} \\
& =g(g-1)^{-1} Z_{j}^{\prime}\left(I_{s}-e e^{\prime} / e^{\prime} e\right) Z_{j}=Z_{j}^{\prime} A Z_{j}
\end{aligned}
$$


observe that $Z_{i j}$ is independent of the elements of matrix $A$ and that:

$M A M A M=M A M$

then, with Ogasawara and Takahashi's theorem cited by Rao (1973, p. 188) we can conclude that the conditional distribution of $S S D_{j}$, given $e_{i}$, is a $\sigma^{2} \chi^{2}$ with $r(A M)=s-2$ d.f. Similarly it can be seen that if $\gamma_{i j}=0$, $g(g-1)^{-1} \Sigma_{i} Z_{i j}^{2}$ follows a $\sigma^{2} \chi^{2}$ with $s-1$ d.f. [this result was obtained by Ellenberg (1977) with the characteristic function technique]. Then if a within $(i, j)$ error estimate of $\sigma^{2}$ is available (let us say $\hat{\sigma}_{0}^{2}$, with $\eta_{0}$ d.f.) we could reject the hypothesis that the additive model is adequate for genotype $j$ if $F_{c} \geq F_{a}$ where:

$F_{c}=g \Sigma_{i} Z_{i j}^{2} /(g-1)(s-1) \hat{\sigma}^{2}$

will be distributed as $F$ on $(s-1)$ and $n_{0}$ degrees of freedom.

On the other hand, we reject the hypothesis that the multiplicative model is adequate for genotype $j$ if $F_{\mathrm{c}} \geq F_{a}$ where

$F_{c}=S S D_{j} /(s-2) \hat{\sigma}_{0}^{2}$.

It is not difficult to verify that these sets of hypotheses are not statistically independent but they can be useful to detect for which genotype the assumed model fails.

Now, if we assume that $\gamma_{i j}$ are random independent normal variables the significance of genotype-environment interaction confirms the presence of the variance component $\sigma_{\gamma}^{2}$. In this case, if it is suspected that genotypes have different 'stability variance' $\sigma_{j}^{2}$ (Shukla, 1972), the hypothesis $H_{0}: \sigma_{j}^{2}=\sigma_{\gamma}^{2}$ can be tested with the procedure given by Ellenberg (1977) in which $H_{0}$, is rejected if

$$
C=\operatorname{Max}_{j} \Sigma_{i} Z_{i j}^{2} / \Sigma_{i j} Z_{i j}^{2}>C_{\alpha}
$$

with a significance level not greater than $\alpha$. If $H_{0}$ is rejected we can use (10) to detect for which genotypes it can be assumed that $\sigma_{j}^{2}=0$.

If we try to model genotype-environment interaction with model (6), but now with the additional assumption that $d_{i j}$ are random normal variables with mean zero and covariance matrix $H \sigma_{r}^{2}$, where $\sigma_{r}^{2}$ can also be termed 'stability variance', test $(11)$ can be used to identify non-stable genotypes.

\section{Examples}

Example 1. In this example we have a case in which the multiplicative model is adequate for explaining a twoway interaction.

In Table 1 we have data for eight bean genotypes in four environments (sowing dates) studied by Valen-
Table 1 Yields of bean genotypes in ton/ha

\begin{tabular}{|c|c|c|c|c|c|}
\hline \multirow[b]{2}{*}{ Genotypes } & \multicolumn{4}{|c|}{ Environments } & \multirow[b]{2}{*}{$b_{j}$} \\
\hline & 1 & 2 & 3 & 4 & \\
\hline 1 & 2.484 & 2.848 & 3.354 & 3.764 & $1.0008^{*}$ \\
\hline 2 & 2.780 & 2.788 & 2.972 & 3.035 & $-0.5677^{*}$ \\
\hline 3 & 2.453 & 3.200 & 3.300 & 3.537 & 0.5423 \\
\hline 4 & 2.720 & 2.753 & 3.385 & 3.690 & $0.6221^{*}$ \\
\hline 5 & 2.265 & 2.512 & 2.937 & 3.234 & 0.5312 \\
\hline 6 & 1.827 & 2.442 & 2.092 & 2.529 & -0.1491 \\
\hline 7 & 2.673 & 2.815 & 2.750 & 3.020 & -0.5275 \\
\hline 8 & 3.332 & 2.917 & 2.889 & 3.000 & $-1.4521^{*}$ \\
\hline$e_{i}$ & -0.317 & -0.100 & 0.075 & 0.342 & \\
\hline
\end{tabular}

$\Sigma b_{j}^{2}=4.69624 .{ }^{*}$ Significant at $\alpha=0.05$.

Table 2 Analysis of variance of bean data

\begin{tabular}{lrrlll}
\hline SV & d.f. & $S S$ & $M S$ & \multicolumn{1}{l}{$F_{c}$} & $F_{a=0.05}$ \\
\hline$E$ & 3 & 7.46332 & 2.48777 & $45.39^{*}$ & 3.86 \\
$R$ & 3 & 0.78565 & 0.26216 & & \\
Error $(A)$ & 9 & 0.49365 & 0.05485 & & \\
$G$ & 7 & 10.54886 & 1.50698 & $19.82^{*}$ & 2.14 \\
$G \times E$ & 21 & 6.19066 & 0.29478 & $3.88^{*}$ & 1.71 \\
$\quad$ Slopes & 7 & 4.38122 & 0.62589 & $4.84^{*}$ & 2.76 \\
$\quad$ Residual & 14 & 1.80944 & 0.12925 & $1.70 \mathrm{~ns}$ & 1.80 \\
Error $(B)$ & 84 & 6.38659 & 0.07603 & & \\
\hline
\end{tabular}

zuela (1985) in a split-plot (sowing dates in whole plots) randomized complete block design with four replicates.

Analysis of variance in units of single plot basis is shown in Table 2. Observe that to include SSS in this table it is multiplied by the number of replicates $r$ and that $S S D R$ in this table is divided by $r$ to test $H_{0}: \beta_{j}=0$.

$$
\begin{aligned}
S S S & =r \Sigma_{j} b_{j}^{2} \Sigma_{i} e_{i}^{2} \\
& =4 \times 4.69624 \times 0.23323=4.38122 .
\end{aligned}
$$

In this calculation $\Sigma_{i} e_{i}^{2}$ is obtained from

$$
\begin{aligned}
r g \Sigma_{i} e_{i}^{2} & =r g e^{\prime} e=S S E(S S \text { of environments }) \\
S S D R & =S S G E-S S S \\
& =6.19066-4.38122=1.80944 .
\end{aligned}
$$

Hypothesis $H_{0}: \beta_{j}=0$ will be rejected at level $\alpha=0.05$ if

$$
\begin{aligned}
\left|b_{j}\right| & \geq t_{\alpha / 2} \sqrt{\left[S S D R /(s-2) \text { rge }^{\prime} e\right]} \\
& =t_{\alpha / 2} \sqrt{[S S D R /(s-2) S S E]} \\
& =2.145 \sqrt{(1.80944 / 2 \times 7.46332)}=0.747 .
\end{aligned}
$$


With this test, only $\beta_{1}$ and $\beta_{8}$ are different from zero at $\alpha=0.05$, but note that SSDR is not significant in Table 2 (the multiplicative model is adequate for explaining the genotype-environmental interaction), a more accurate conditional estimate of the error component is possible by pooling $S S D R$ and the $S S$ of Error. If this is done, $H_{0}: \beta_{j}=0$ will be rejected at the level $\alpha=0.05$ if

$$
\begin{aligned}
\left|b_{j}\right| & \geq t_{\alpha / 2} \sqrt{\left[(g-1) \hat{\sigma}^{2} / \text { rge }^{\top} e\right]} \\
& =t_{\alpha / 2} \sqrt{\left[(g-1) \hat{\sigma}^{2} / S S E\right]} \\
& =1.99 \sqrt{(7 \times 0.08363 / 7.46332)}=0.557
\end{aligned}
$$

where $\hat{\sigma}^{2}=(S S D R+S S$ Error $) / 98=0.088363$.

So, $\beta_{1}, \beta_{2}, \beta_{4}$ and $\beta_{8}$ are declared different from zero at the level $\alpha=0.05$ (Table 1).

Coefficients $\beta_{j}$ could be interpreted as a measure of sensitivity Talbot (1982). For example, genotype 1 has better performance in environments with high yield production (environments 3 and 4 ) while genotype 8 has better performance in environments with low yield production (environment 1).

Example 2. In this example we will consider the classical barley data analysed by Yates \& Cochran (1938). We shall only consider the part of the table dealing with genotype-environment interaction. In Table 3, the genotype $\times$ environment totals are given and Table 4 shows that genotype-environment interaction is significant. In order to test $H_{0}: \sigma_{j}^{2}=\sigma_{\gamma}^{2}$ we get

$C=750.47 / 1477.69=0.5078$.

As $C_{0.05}=0.4824$ (table 1 given in Ellenberg, 1977), we reject $H_{0}$ and with test $(10)$ we conclude that genotypes 4 and 5 are unstable. Further regression analysis shows that the multiplicative model is significant at level $\alpha=0.05$ but SSDR is also significant. Hypothesis $H_{0}: \beta_{j}=0$ will be rejected at the level $\alpha=0.05$ if

$$
\begin{aligned}
\left|b_{j}\right| & \geq t_{\alpha / 2} \sqrt{[S S D R /(s-2) S S E]} \\
& =2.12 \sqrt{(704.69 / 4 \times 7072.92)}=0.335 .
\end{aligned}
$$

In Table 3 it can be seen that regression coefficients of genotypes 4 and 5 are different from zero at level $\alpha=0.05$. In order to investigate whether the multiplicative model fails just for one or two genotypes, $S S D R$ is split in sums of squares, one for each genotype. In Table 4 we can see that genotype 4 remains unstable, although its variability has reduced considerably. Similar conclusions were drawn by Yates \& Cochran (1938) and Shukla (1972) but the above type of analysis uses exact conditional tests and so the levels of significance are exact.

Note that sums of squares are divided by 6 , $M S R G_{j}=g S S R G_{j} /(g-1)(s-1)$ and $M S D R_{j}=g S S D R_{j} /$ $(g-1)(s-2)$.

\begin{tabular}{|c|c|c|c|c|c|c|c|}
\hline \multirow[b]{2}{*}{ Genotypes } & \multicolumn{7}{|c|}{ Environments } \\
\hline & 1 & 2 & 3 & 4 & 5 & 6 & $b_{j}$ \\
\hline 1 & 161.7 & 247.0 & 185.4 & 218.7 & 165.3 & 154.6 & -0.156 \\
\hline 2 & 187.7 & 257.5 & 182.4 & 183.3 & 138.9 & 143.8 & -0.014 \\
\hline 3 & 200.1 & 262.9 & 194.9 & 220.2 & 165.8 & 146.3 & -0.054 \\
\hline 4 & 196.9 & 339.2 & 271.2 & 266.3 & 151.2 & 193.6 & $0.609^{*}$ \\
\hline 5 & 182.5 & 253.8 & 219.2 & 200.5 & 184.4 & 190.1 & $-0.385^{*}$ \\
\hline
\end{tabular}

Table 4 Analysis of variance of barley data

\begin{tabular}{lrlrll}
\hline SV & d.f. & \multicolumn{1}{l}{$S S$} & \multicolumn{1}{l}{$M S$} & \multicolumn{1}{l}{$F_{c}$} & $F_{\alpha=0.05}$ \\
\hline$E$ & 5 & 7072.92 & & & \\
$G$ & 4 & 1770.28 & & & \\
$G \times E$ & 20 & 1477.84 & 73.89 & $3.17^{*}$ & 1.57 \\
$R G_{j}$ & & & & & \\
1 & 5 & 151.52 & 37.88 & 1.63 & 2.37 \\
2 & 5 & 132.69 & 33.17 & 1.42 & \\
3 & 5 & 142.08 & 35.52 & 1.53 & \\
4 & 5 & 750.47 & 187.62 & $8.06^{*}$ & \\
5 & 5 & 300.93 & 75.23 & $3.23^{*}$ & \\
& 4 & 773.16 & 193.29 & $4.39^{*}$ & 3.01 \\
Slopes & 4 & 704.69 & 44.04 & $1.89^{*}$ & 1.47 \\
Res. & 16 & & & & \\
$D R_{j}$ & & & & & \\
1 & 4 & 117.10 & 36.59 & 1.57 & 2.21 \\
2 & 4 & 132.41 & 41.38 & 1.78 & \\
3 & 4 & 137.96 & 43.11 & 1.85 & \\
4 & 4 & 225.78 & 70.56 & $3.03^{*}$ & \\
5 & 4 & 91.23 & 28.51 & 1.22 & \\
$\sigma^{2}$ & 216 & & 23.28 & & \\
\hline
\end{tabular}

*Significant at $\alpha=0.05$.

Table 3 Genotype $\times$ environment totals of barley data

*Significant at $\alpha=0.05$. 


\section{Discussion and conclusions}

Some exact conditional tests have been derived assuming that interaction is not present. In a typical genotype trial as the complete block design is used in several places we have an estimate of the within $(i, j)$ cell error so that an exact genotype-environment interaction test is available. If the interaction is significant then the proposed tests fail. Nevertheless, these tests remain valid if we assume that model (6) is correct but now with the additional assumption that $d_{i j}$ are random normal variables with mean zero and covariance matrix $H \sigma_{r}^{2}$ where $\sigma_{r}^{2}$ can be termed the 'stability variance' (Shukla, 1972). If it is suggested that genotypes have different 'stability variance' say $\sigma_{j}^{2}$, test $(10)$ can be used to identify non-stable genotypes.

\section{References}

EBERHART, S. A. AND RUSSELL, w. A. 1966. Stability parameters for comparing varieties. Crop Sci., 6, 36-40.

ELLENBERG, J. H. 1977. The joint distribution of the standardized row sum of squares from a balanced two-way layout. J. Am. Stat. Assoc., 72, 407-411.

GABRIEL, K. R. 1978. Least squares approximation of matrices by additive and multiplicative models. J. Roy. Stat. Soc., Series $B, 2,186-196$.

MANDEL, J. 1961. Non-additivity in two-way analysis of variance. J. Am. Stat. Assoc., 56, 878-888.

MILLIKEN, G. A. AND GRAYBILL, F. A. 1970. Extensions of the general linear hypothesis model. J. Am. Stat. Assoc., 65, 797-807.

RAO, C. R. 1973. Linear Statistical Inference and its Applications. John Wiley and Sons, Inc., Chichester.

SCHEFFE, H. 1959. The Analysis of Variance. John Wiley and Sons, Inc., Chichester.

SHUKLA, G. L. 1972. Some statistical aspects of partitioning environmental components of variability. Heredity, 23 , 237-245.

TAlbot, M. 1982. Variability in Herbage Trials. M. Phil thesis, University of Edinburgh.

TUKEY, J. w. 1949. One degree of freedom of non-additivity. Biometrics, 5, 232-242.

VAlenzuela, L. J. 1985. Parámetros de estabilidad para el rendimiento de variedades de frijol (Phaseolus vulgaris L.) en cuatro fechas de siembra. Agric. Téc. México, 11, $185-200$.

WESTCOTT, в. 1986. Some methods of analysing genotypeenvironment interaction. Heredity, 56, 243-253.

YATES, F. AND COCHRAN, W. G. 1938. The analysis of groups of experiments. J. Agric. Sci., 28, 556-580.

\section{Appendix}

Derivation of the conditional covariance matrix of $\beta$ given $e_{i}$.
If we define $B^{\prime}=\left(\beta_{1}, \beta_{2}, \ldots, \beta_{g-1}\right)$, it can be seen that $\beta=C B$; where $C$ is a matrix of order $g(g-1)$ defined by

$C^{\prime}=\left(I_{g-1},-1_{g-1}\right)$.

It can be proved that $b^{\prime}=\left(b_{1}, b_{2}, \ldots, b_{g-1}\right)$ where $b_{j}$ is defined in (4) is a linear combination of $Z=H Y$ because

$$
\begin{aligned}
b & =\left[(e \otimes C)^{\prime}(e \otimes C)\right]^{-1}(e \otimes C)^{\prime} Z \\
& =\left(1 / e^{\prime} e\left(\left(C^{\prime} C\right)^{-1}\left(e^{\prime} \otimes C^{\prime}\right) Z .\right.\right.
\end{aligned}
$$

Now observe that $C^{\prime} C=I_{g-1}+J_{g-1}$ is a patterned matrix Graybill $(1969$, p. 171$)$ and $\left(C^{\prime} C\right)^{-1}=$ $(1 / g)\left(g I_{g-1}-J_{g-1}\right)$ so that

$$
b=\left(1 / g e^{\prime} e\right)\left(g I_{g-1}-J_{g-1}\right)\left(e^{\prime} \otimes C^{\prime}\right) Z \text {. }
$$

Since

$$
\begin{aligned}
{\left[\left(e^{\prime} \otimes C^{\prime}\right) Z\right]^{\prime}=} & {\left[\left(e_{1} C^{\prime}, e_{2} C^{\prime}, \ldots, e_{s} C^{\prime}\right\rangle Z\right]^{\prime} } \\
= & {\left[\Sigma e_{i}\left(Z_{i 1}-Z_{i g}\right), \Sigma e_{i}\left(Z_{i 2}-Z_{i g}\right), \ldots,\right.} \\
& \left.\Sigma e_{i}\left(Z_{i(g-1)}-Z_{i g}\right)\right]
\end{aligned}
$$

then

$b_{j}=\left(1 / g e^{\prime} e\right)\left[g \Sigma_{i} e_{i}\left(Z_{i j}-Z_{i g}\right)-\sum_{j=1}^{g-1} \Sigma_{i} e_{i}\left(Z_{i j}-Z_{i g}\right)\right]$

but

$\sum_{j=1}^{g-1}\left(Z_{i j}-Z_{i g}\right)=-g Z_{i g}$

and we obtain

$$
\begin{aligned}
b_{j} & =\left(1 / g e^{\prime} e\right)\left[g \Sigma_{i} e_{i}\left(Z_{i j}-Z_{i g}\right)+g \Sigma_{i} e_{i} Z_{i g}\right] \\
& =\left(1 / e^{\prime} e\right) \Sigma_{i} e_{i} Z_{i j} .
\end{aligned}
$$

Using the properties of linear combinations of normal vectors we get

$\operatorname{Cov}(b)=\left[1 /\left(e^{\prime} e\right)^{2}\right]\left(C^{\prime} C\right)^{-1}\left(e^{\prime} \otimes C^{\prime}\right) H(e \otimes C)\left(C^{\prime} C\right)^{-1} \sigma^{2}$ and from some properties of the direct product of matrices it can be shown that

$\operatorname{Cov}(b)=\left(1 / e^{\prime} e\right)\left(C^{\prime} C\right)^{-1} \sigma^{2}=\left(1 / g e^{\prime} e\right)\left(g I_{g-1}-J_{g-1}\right) \sigma^{2}$ where $\operatorname{Cov}(b)$ stands for the covariance matrix of vector $b$, and as:

$\hat{\beta}^{\prime} C b$ it can be seen that

$\operatorname{Cov}(\hat{\beta})=\left(1 / g e^{\prime} e\right)\left(g I_{g}-J_{g}\right) \sigma^{2}$

is the conditional covariance matrix of vector $\hat{\beta}$ given $e_{i}$. 